D O S S I E R

Genèses 56, sept. 2004, pp. 28-50
D'UN MARCHÉ

NATIONAL DES

\section{FRUITS ET LÉGUMES :}

ENTRE ÉCONOMIE,

\section{Antoine Bernard de Raymond}

1. «La Vallée» est le nom que l'on donne alors au marché de la volaille et du gibier à la halle de Paris, situé, avant la construction des halles Baltard, quai de la Mégisserie, surnommé «Vallée de la misère». Après l'installation du marché dans un des pavillons nouvellement édifiés, on différencie l'actuel et le précédent site en les appelant respectivement «nouvelle» et «ancienne Vallée». Le toponyme «Vallée de la misère» en lui-même correspond à l'espace fluvial entre le pont au change et le pont Notre-Dame, et il rappelle les nombreux accidents liés à la difficulté de franchir successivement les deux ponts dont les deux arches ne sont pas alignées.
$\mathrm{E}$ $\mathrm{n}$ avril et mai 1876 se tiennent, aux assises de la Seine, les deux procès dits «de la Vallée» ${ }^{1}$, que l'on nomme aussi «l'affaire des halles». À cette occasion éclate au grand jour la pratique généralisée de la corruption sur le marché de la volaille et du gibier. Lors du premier procès sont jugés les délits commis sur le marché à l'amiable, où les marchands forains réalisent eux-mêmes les ventes. Mais c'est sans doute le second procès, celui de mai 1876 , où seront jugées les fraudes commises sur le marché à la criée, qui aura la plus grande signification.

Lors de ce deuxième procès sont inculpés uniquement des agents de la préfecture de Police et de la préfecture de la Seine, soupçonnés de s'être rendus coupables de fraude, d'abus de confiance et de vols. Le marché à la criée sert à vendre les marchandises des éleveurs et expéditeurs de province qui ne peuvent se rendre eux-mêmes à la halle pour y commercialiser leurs produits. Ce sont ceux-là mêmes qui sont censés vendre ces produits au nom des éleveurs et expéditeurs provinciaux qui les ont trompés.

Ainsi, alors que le premier procès traite somme toute d'une banale affaire de corruption, le second met en question le fonctionnement même du marché parisien et les relations qu'il entretient avec la province: la croissance de la population de la capitale et l'augmentation progressive des salaires réels dans la seconde moitié du $\mathrm{XIX}^{\mathrm{e}}$ siècle 
exercent une forte pression sur l'offre de produits alimentaires, qui y répond par un élargissement de ses zones d'approvisionnement.

À mesure que se généralise la pratique du commerce à distance, le problème de la représentation des éleveurs, producteurs et expéditeurs de province devient sans cesse plus épineux. Lors du premier procès, les défenseurs des marchands forains inculpés ont réclamé à cor et à cri qu'on rétablisse le principe de la liberté de commerce, la lourdeur des contrôles administratifs étant selon eux responsable de la fraude. Mais il s'agit là de l'argument de marchands qui vendent eux-mêmes les denrées qu'ils ont envoyées vers Paris. Les agriculteurs et les expéditeurs de province voient eux d'un très mauvais œil la possibilité offerte par décret en 1878 aux facteurs ${ }^{2}$ de pratiquer la vente pour leur propre compte, car ils n'ont dès lors plus aucun moyen de s'informer de ce qu'il advient des fruits de leur travail.

Face aux plaintes répétées adressées au Parlement par les sociétés d'agriculture à propos des abus dont les producteurs de province continuent à être victimes aux halles centrales de Paris, une commission parlementaire est nommée en 1891, qui élaborera une proposition de loi tendant à y instaurer une nouvelle réglementation.

Les débats parlementaires que suscite cette proposition de loi font apparaître - outre une opposition classique entre les partisans d'une régulation administrative des transactions et ceux qui voient dans le principe de marché la meilleure garantie de la sincérité des transactions - une difficulté majeure, qui réside dans la détermination de la portée (dans l'espace physique) de la loi. Mais ce problème n'est qu'effleuré par les députés et sénateurs français lors des débats sur la proposition de loi, et les parlementaires se focalisent avant tout sur la définition des fonctions des individus autorisés à opérer des ventes aux halles et les contrôles administratifs qui y seront liés. Aussi, et peut-être surtout, cette question de la validité spatiale de la loi est laissée de côté en partie parce qu'on ne voit que trop bien qu'elle serait source de trop de confits entre les uns et les autres (libéraux et partisans des contrôles administratifs, élus parisiens et élus des circonscriptions rurales, élus proches des producteurs et élus proches des commerçants, etc.).

En tout état de cause, cette incertitude quant à la signification de la loi va en générer des interprétations divergentes. Elle va en outre permettre une transformation
2. Le facteur est un intermédiaire officiel des ventes, qui, effectuant des transactions pour le compte d'autrui, est censé garantir leur loyauté. Le décret de 1878 modifie cette situation de principe, en introduisant pour le facteur la possibilité de vendre comme commissionnaire. 


\section{O S S I E R}

Espaces de l'activité économique

Antoine Bernard de Raymond La construction d'un marché national des fruits et légumes: entre économie, espace et droit (I896-I995)
3. Max Weber, Wirtschaft und Gesellschaft, Tübingen, Mohr, 1956 et Rechtssoziologie, Neuwied am Rhein, Luchterhand, 1967, (trad. fr., Économie et société, t. 2, Paris, Plon, 1971, p. 11).

4. Ibid. du marché qui n'était pas du tout celle attendue par le législateur et poser des problèmes qui, même s'ils se présentent sous un jour différent désormais, sont toujours «d'actualité». En effet, c'est en partie sur l'échec de la loi du 11 juin 1896 que se bâtit la politique des Marchés d'intérêt national (MIN), à partir de 1953, lesquels marchés servent encore aujourd'hui à la commercialisation des fruits et légumes frais. Si, dans son intention, cette politique se démarque fortement de la loi de 1896, dans la mesure où elle vise non pas à organiser le marché parisien - conçu comme centre du dispositif français de distribution des fruits et légumes - mais au contraire à rationaliser les circuits commerciaux sur l'ensemble du territoire français (et, justement, éviter que soient expédiées «à l'aveugle» la plupart des marchandises vers la capitale), elle vise en revanche explicitement à dépasser les apories de la loi de 1896, dues à l'absence de définition précise du marché, en instituant autour des MIN des périmètres de protection à l'intérieur desquels il est interdit de pratiquer le commerce de gros de denrées alimentaires. Mais là encore, on verra que la mise en œuvre de la loi révèlera des situations économiques qui n'avaient pas clairement été envisagées par le législateur, les MIN ayant finalement une portée empirique à l'opposé de la fonction qui leur avait initialement été attribuée: au lieu d'être le lieu de la modernisation de la distribution des denrées alimentaires, les MIN en viennent à jouer le rôle d'espaces de protection du commerce traditionnel.

Le propos de cet article n'est en aucune manière de stigmatiser l'incurie des pouvoirs publics, ni de critiquer «l'incomplétude» des règles de droit qu'ils ont produites. Le fait de voir dans la complétude des contrats (ou des règles de droit) une solution optimale à l'incertitude à laquelle peuvent faire face les acteurs économiques reflète une incompréhension profonde des rapports entre le droit et le «social». En effet, une telle conception (que fait sienne une part importante du courant de recherche dit de «l'économie du droit») repose sur un double implicite: d'abord elle oppose arbitrairement normes et marché, les faisant apparaître comme deux sphères totalement hétérogènes; ensuite elle adopte le «point de vue juridique ${ }^{3}$ » sur le droit, qui voit dans la norme une «injonction à faire».

Le «point de vue sociologique» sur le droit consiste à considérer «ce qu'il en advient en fait dans la communauté ${ }^{4} », c^{\prime}$ est-à-dire comment les acteurs sociaux se servent 
des normes (règles de droit, contrats) comme de repères pour fixer leurs anticipations, orienter leurs activités 5 . De ce point de vue, les divergences d'interprétation des règles de droit n'ont rien que de très normal, et la règle de droit est par nature incomplète. Comme le fait remarquer Claude Didry, «l'ambivalence est [...] constitutive de la nature de la règle de droit, elle correspond à sa "texture ouverte" ${ }^{»}$. Dès lors deux conclusions s'imposent: (1) normes et marché ne sont pas antagoniques, mais le marché (dans ses structures et son fonctionnement) est directement informé par les normes, et (2) les effets d'une règle de droit sur l'activité économique ne peuvent se déduire a priori mais seulement de l'étude empirique de la signification que les agents lui donnent. Cette mobilisation de la règle de droit par les agents entraîne invariablement une modification de la portée de cette dernière (par rapport à son sens « dogmatique $\left.{ }^{7} »\right)$.

Ainsi on est conduit à envisager les rapports entre économie et action publique comme une relation dialectique: les normes édictées par les pouvoirs publics influent sur l'activité économique au sens où elles constituent un repère pour les agents économiques, mais en retour, la façon dont ces agents s'appuient sur ces normes en modifie la signification et la portée, ce qui conduit souvent à une nouvelle intervention des pouvoirs publics. Il faut donc aussi bien étudier les processus de production des règles de droit que leur mise en œuvre. Car la relation qu'entretien l'action publique avec l'économie n'est pas une relation d'extériorité. Au contraire, l'action publique se situe au cœur de l'activité économique, par la présence de règles de droit en permanence mobilisables par les acteurs économiques.

Je me propose ici, dans une perspective historique, de rendre compte de cette dialectique de l'action publique et de l'économie. Pour cela j'étudierai à la fois les objectifs des pouvoirs publics (qui sont somme toute les mêmes tout au long du siècle, à savoir l'organisation d'une «démocratie représentative de marché»), la logique interne des normes qu'ils produisent, et la mobilisation de ces normes par les acteurs du commerce en fruits et légumes. On verra que la qualification institutionnelle de l'espace joue un rôle décisif dans le conditionnement des stratégies des acteurs économiques vis-à-vis des contrôles administratifs définis par les pouvoirs publics. Autrement dit, si l'espace constitue une dimension importante de l'activité économique, on ne saurait en rendre compte si
5. Voir Alessandro Stanziani, «Action économique et contentieux judiciaires », Genèses, $\mathrm{n}^{\circ}$ 50, 2003, p. 89; Antoine Bernard de Raymond, «La règle de droit comme maxime empirique de l'activité économique»,

Terrains et travaux, $\mathrm{n}^{\circ}$ 6, 2004, pp. 71-80.

6. Claude Didry, «Règle de droit et travail juridique», in Thierry Kirat et Évelyne Serverin (éd.), Le droit dans l'action économique, Paris, CNRS, 2000, p. 134.

7. Max Weber, «R. Stammlers "Überwindung" der materialistischen Geschichtsauffassung», Archiv für Sozialwissenschaft und Sozialpolitik, vol. 24, n 1, 1907, pp. 94-151 (trad. fr., Rudolf Stammler et le matérialisme historique, 1907, Québec, Presses de l'université de Laval, Paris, Cerf, 2001, p. 133). 


\section{O S S I E R}

Espaces de l'activité économique

Antoine Bernard de Raymond La construction d'un marché national des fruits et légumes: entre économie, espace et droit (I896-1995)
8. Journal officiel de la République française $(J O R F)$.

Débats parlementaires, Sénat, 18 février 1895, intervention de Paul Le Breton (sénateur de la Mayenne, monarchiste). l'on considère l'espace d'un point de vue purement physique, alors que ses déterminants sont multiples, en particuliers légaux et judiciaires.

\section{Réglementer les halles: la loi du 11 juin 1896}

L'exaspération des producteurs et expéditeurs de province face aux abus dont ils sont victimes aux halles centrales de Paris entraîne, comme on l'a vu, la création d'une commission parlementaire, dirigée par le député Gustave Cluseret. Ladite commission avance une proposition de loi tendant à la réglementation des halles centrales de Paris. Cette proposition de loi sera discutée pendant trois ans, pour être finalement adoptée le 11 juin 1896.

L'intervention du Parlement a pour but d'assurer la loyauté des transactions aux halles, c'est-à-dire de mettre fin aux «abus» et à la fraude qui y règnent. Si l'on a jugé nécessaire le vote d'une loi c'est parce que la région parisienne n'est pas autosuffisante en matière d'alimentation, et que de plus en plus de produits de toutes les régions de France parviennent aux halles centrales de Paris et qu'en conséquence la réglementation du marché parisien concerne la nation dans son ensemble. Mais avant d'examiner la façon dont les parlementaires envisagent de réguler le marché, il faut d'abord déterminer quelles sont les causes que ceux-ci identifient, parfois de manière contradictoire, comme étant à l'origine des désordres constatés.

Les deux catégories de fraudes les plus fréquemment mentionnées, à propos des halles centrales, sont le regrat et la resserre. Le regrat consiste à réaliser une vente fictive d'un certain lot de denrées:

«On présente le lot et, sans que personne ne se soit présenté pour l'acheter, on le porte comme vendu à un prix imaginaire, on le fait disparaître, et, quelques instants après, on le représente de nouveau aux acheteurs réels pour le vendre à un prix supérieur ${ }^{8}$.»

Ensuite, le grossiste indique à son fournisseur le prix de la vente fictive, et garde pour lui la marge entre le premier prix fictif et le prix de la vente réelle qu'il a ensuite réalisée. Si une telle manœuvre est possible, c'est parce que le décret de 1878 prétendait instituer un type d'acteur unique sur le marché des halles, le facteur, qui n'est pas autorisé à réaliser des opérations commerciales en son nom propre, mais sans pour autant interdire formellement les actes de commerce (si le vendeur se porte 
«commissionnaire» au lieu de «facteur»). Ainsi le facteur peut «faire la contrepartie», c'est-à-dire vendre à lui-même un lot de manière fictive pour le compte de l'expéditeur puis le vendre une seconde fois, à un prix plus élevé, cette fois-ci pour son propre compte. La resserre consiste à cacher une partie des stocks réellement détenus pour laisser penser aux acheteurs que l'offre est plus réduite qu'elle ne l'est réellement. Ceci permet de maintenir les cours à un niveau relativement élevé lorsque la marchandise est trop abondante. Si, lorsqu'ils remettent le lot en vente le lendemain, les vendeurs jugent les prix toujours trop faibles, ils demandent aux agents de l'administration de saisir les denrées, sous prétexte qu'elles sont avariées. Ainsi cette pratique lèse à la fois le consommateur et le producteur.

Pour les uns, le renforcement des contrôles administratifs permettra seul de faire cesser les pratiques frauduleuses, ce qui implique de donner un statut particulier aux grossistes. Pour les autres au contraire, l'attribution d'un tel statut aux grossistes reviendrait à constituer un monopole au profit d'un corps de vendeurs, qui seraient à même de se livrer sans retenue à toutes sortes de pratiques monopolistiques. Pour eux il importe au moins de préserver, à côté du marché officiel, un marché libre qui, par la concurrence qui y règnera, entraînera l'élimination de tous les intermédiaires malhonnêtes ${ }^{9}$.

La difficulté réside en ce que les termes de ce débat entre contrôles administratifs et responsabilité individuelle changent selon la région d'origine des produits. En effet, l'argument qui veut que les intermédiaires malhonnêtes perdront peu à peu leurs fournisseurs et seront tôt ou tard éliminés du marché ne tient pas pour des expéditeurs ou des producteurs situés dans des régions éloignées, d'où il est difficile de s'assurer du devenir des produits envoyés à Paris. Cet élargissement géographique du marché, permis par l'amélioration des techniques de conservation et de transport (avec en particulier le développement du chemin de fer) à la fin du XIX ${ }^{\mathrm{e}}$ siècle, s'il génère de nouveaux débouchés pour les agriculteurs français, est aussi vecteur d'incertitude quant aux conditions de la vente des biens, dont les intermédiaires parisiens peuvent être tentés de tirer profit ${ }^{10}$.

$\mathrm{Si}$, dans l'absolu, tout le monde convient de ce qu'il faut réduire au strict minimum le nombre d'intermédiaires et qu'une solution optimale consisterait à permettre aux «propriétaires » ${ }^{11}$ de vendre eux-mêmes leurs
9. On trouve une illustration de cette opposition dans la discussion suivante, où le rapporteur de la proposition de loi - Jean Camescasse (sénateur du Pas-deCalais, Gauche républicaine) - va être interrompu par un partisan de la dualité des statuts sur le marché:

«M. le rapporteur. Il y a [...] un agent officiel, le facteur, qui est cautionné et surveillé; et puis nous avons cet intermédiaire qui s'appelle le commissionnaire. Celui-ci n'offre comme garantie que sa moralité et la situation qu'une clientèle souvent ancienne lui a faite peu à peu [...]

Quelle est la conséquence

de cette dualité des vendeurs?

M. Buffet. C'est de respecter le libre choix des expéditeurs.

- C'est facile à dire [...].

Si le commissionnaire ne présente pas une garantie suffisante..

- Cela le regarde!

- Il est certain que quand une personne habite à cinquante lieues et qu'elle a besoin d'envoyer sa marchandise aux Halles, parce que c'est le lieu de rassemblement naturel, elle ne sait pas à qui s'adresser. - Elle peut s'adresser aux facteurs si elle n'a pas confiance dans les commissionnaires. » (JORF. Débats parlementaires, Sénat, 18 février 1895).

10. Comme le fait remarquer Étienne Ladoucette (député des Ardennes, bonapartiste, puis proche des monarchistes), dans un rapport qui inspira la proposition de loi déposée par Gustave Cluseret (député du Var, apparenté socialiste): «[Des producteurs] ont lu une belle circulaire d'un commissionnaire aux halles centrales dont le titre est habilement confondu avec celui de facteur. Sur la foi de ces promesses, ils ont envoyé leurs produits à cet intermédiaire et ils attendent en vain le résultat de la vente. La réponse tant attendue n'arrive qu'après bien des réclamations et leur apprend que le prix de vente est inadmissible» (JORF. Débats parlementaires, Chambre des députés, 26 juin 1893).

\section{Quand il est question} de «propriétaires », les parlementaires pensent implicitement aux producteurs. Mais ces deux catégories ne se recouvrent pas forcément. Cette ambiguïté dans l'usage du terme "propriétaire», notamment dans le texte final de la loi, sera source 


\section{O S S I E R}

Espaces de l'activité économique

Antoine Bernard de Raymond La construction d'un marché national des fruits et légumes: entre économie, espace et droit (I896-I995)

d'énormes déconvenues pour les tenants d'un encadrement administratif strict du marché.

\section{L'expression est ici employée} telle qu'elle l'était dans le sens commun à l'époque, alors que la signification juridique du terme est sensiblement différente. Là encore, on rencontre une ambiguïté qui affectera de manière importante le développement du marché.

13. JORF. Débats parlementaires, Sénat, 18 février 1895. Intervention de M. Le Breton.

14. JORF. Débats parlementaires, Sénat, 18 février 1895. marchandises, les avis divergent fortement quant à la façon d'appliquer concrètement ce principe. Il existe toutefois une catégorie de producteurs qui peuvent à la fois remplir les fonctions de producteur et de vendeur aux «halles centrales ${ }^{12} »$, ce sont les producteurs maraîchers de la région parisienne. Un secteur des halles leur est traditionnellement réservé depuis une délibération du conseil municipal de la ville de Paris du 13 mai 1880 autorisant la vente au détail des denrées végétales sur le secteur dit du «carreau». Ainsi, ces producteurs doivent se limiter à la vente de détail des denrées qu'ils apportent eux-mêmes sur le marché.

Mais pour les producteurs provinciaux, il est évident qu'il faudra trouver une autre solution. Pour les uns, il faut s'en tenir au «droit commun de tous les Français», c'est-à-dire à la liberté de commerce:

«[...] le plus simple consiste à permettre au producteur de vendre lui-même sa marchandise, et, s'il ne peut venir en personne, de choisir l'homme dans lequel il aura assez confiance pour le charger de cette opération ${ }^{13}$ ».

Autrement dit, il s'agit de mettre en place des structures de commercialisation propres aux producteurs, au sein desquelles les éventuels intermédiaires seraient contrôlés par ces derniers, et donc contraints de se comporter de manière loyale. Mais l'argument est rejeté par le commissaire du gouvernement qui répond:

«Introduire le propriétaire aux Halles c'est l'idéal en théorie, mais en fait il y a un malheur: c'est que nous aurons beau l'appeler, il ne répondra jamais à notre appel ${ }^{14}$.»

Ainsi, c'est vers l'organisation de la représentation des producteurs que s'oriente la loi. Si l'on refuse la présence du «propriétaire», se pose alors la question de savoir comment l'on s'y prendra pour informer celui-ci du devenir des «efforts de son travail», autrement dit, en quoi consistera le contrôle administratif supposé mettre fin aux fraudes que le seul droit commun ne permet pas d'empêcher. Dans ce but, la loi dispose que chaque vente devra figurer dans la comptabilité du vendeur et être consignée sur deux feuilles de vente, l'une accompagnant le lot vendu et l'autre étant envoyée à l'expéditeur. Tout colis vendu devra immédiatement quitter les halles (afin d'empêcher les ventes fictives successives). La responsabilité des contrôles sera partagée entre la préfecture de Police, qui assure le maintien du bon ordre au point de vue de la loyauté des transactions, de la salubrité des 
denrées et de la liberté de circulation, et la préfecture de la Seine qui s'occupera de la répartition des emplacements entre les différents vendeurs. Les vendeurs ne seront pas propriétaires de leurs emplacements, mais ceux-ci leur seront attribués sous le régime de la concession, autrement dit ces emplacements seront à tout moment révocables. Le point central (du point de vue «dogmatique») du texte de loi est constitué par la définition des modes ventes autorisés et du statut des vendeurs. On l'a vu, l'objectif du législateur était d'empêcher les ventes fictives. Pour cela, la loi interdit aux vendeurs de pratiquer le commerce pour leur propre compte - c'est-àdire que les vendeurs ne peuvent que pratiquer les ventes à la commission pour le compte d'un expéditeur ou d'un producteur. En outre on retient le principe d'un statut unique pour les vendeurs, celui de «mandataire» (articles 3 et 4), soumis à un cautionnement et à des contrôles administratifs, et opérant (cela est d'importance pour la suite de mon propos) à l'intérieur des pavillons des halles.

En apparence la loi opte de manière claire pour une organisation du marché selon le principe d'une représentation contrôlée par opposition au diptyque propriété et responsabilité individuelle. Mais on va à présent constater que le texte de loi est chargé d'ambiguïtés et d'imprécisions (résultant notamment des compromis qu'il a été nécessaire de passer avec les députés libéraux pour que la loi puisse être adoptée), qui vont peser lourdement sur les possibilités «d'application» de la loi.

\section{La mise en œuvre de la loi: mais où commence et où s'arrête le marché?}

L'économie générale de la loi telle que la conçoit le législateur est somme toute assez simple: il s'agit, pour mettre fin à toutes les fraudes et pratiques monopolistiques constatées aux halles centrales de Paris d'interdire qu'aux halles quiconque pratique la vente en gros de denrées alimentaires pour son propre compte. Pour cela il faut constituer deux privilèges: d'abord, sous les pavillons, au bénéfice des mandataires, qui ne peuvent vendre que la marchandise pour laquelle ils ont reçu le mandat de vendre, et dont l'activité doit être contrôlée par l'administration; ensuite, sur le carreau, aux cultivateurs vendant eux-mêmes le produit de leur récolte. Le contrôle administratif vise à assurer que le marché parisien demeure un marché de première main. Pour cela, 


\section{O S S I E R}

Espaces de l'activité économique

Antoine Bernard de Raymond La construction d'un marché national des fruits et légumes: entre économie, espace et droit (I896-I995)

15. Marthe Torre-Schaub, Essai sur la construction juridique de la catégorie de marché, Paris, LGDJ, 2002, p. 73.

16. Ceci est d'autant plus surprenant que cette incertitude avait été parfaitement identifiée lors du débat au Sénat. Voir JORF.

Débats parlementaires, Sénat, séance du 4 mars 1895.

17. Voir M. Weber, Rudolf Stammler..., op. cit. On peut dire que tel ou tel élément de la réalité sociale a une «signification causale» dans la mesure où des agents lui attribuent un «sens» en fonction duquel ils déterminent leur activité empirique. l'activité des mandataires est enserrée dans toute une série de contrôles. Le problème est que pour que la loi puisse être adoptée, les parlementaires français ont dû opter pour une solution «transactionnelle» et accepter certains compromis. De ce point de vue, un élément en particulier va s'avérer déterminant.

Il subsiste en effet une forte ambiguïté sur la définition des halles. Juridiquement, cette définition est très précise: le terme de «halle» désigne un marché couvert ${ }^{15}$, et devrait donc, en toute logique (juridique), s'appliquer aux seuls pavillons du marché parisien. Mais en réalité, le marché parisien est constitué par ce qu'on appelle le «périmètre des halles », qui englobe les pavillons et le carreau, mais dont la superficie est beaucoup plus importante que celle de ces deux zones. Ainsi le commerce de gros en denrées alimentaires dans Paris ne se fait pas uniquement sous les pavillons Baltard. Or le texte de la loi du 11 juin 1896 ne porte pas trace d'un choix clair entre les deux acceptions du terme, ce qui entraîne parfois des ambiguïtés quant à la zone du marché qui est visée ${ }^{16}$.

Ainsi dans l'article premier, lorsque les halles centrales sont définies comme «un marché de première main [...] des denrées alimentaires de gros et de demi-gros », et qu'à «titre transitoire, quelques pavillons resteront réservés à la vente au détail», on doit comprendre que les halles sont assimilées aux pavillons. Mais quand, dans le même article, il est stipulé que «le carreau est réservé, dans le périmètre des Halles, aux propriétaires de légumes et fruits vendant leur propre marchandise», on a affaire à une conception extensive du marché. Ainsi, la loi, prise dans son sens dogmatique, institue, de manière positive, deux sous-espaces: les pavillons et le carreau. Mais, elle en constitue négativement un troisième, la zone, à l'intérieur du périmètre des halles, qui ne relève ni du carreau ni des pavillons, et où le commerce est, implicitement, placé sous le régime du droit commun.

\section{Marché «officiel » et marché «libre»}

Cette ambiguïté va déterminer de manière décisive la «signification causale ${ }^{17}$ » de la loi du 11 juin 1896 . En effet, en dehors de la zone des pavillons et du carreau, mais à l'intérieur du "périmètre des halles », des commerçants vendent des fruits et légumes (ou d'autres produits), en général en gros et à la commission. La présence des «commissionnaires libres» en dehors de l'espace d'application des contrôles définis par la loi du 11 juin 1896 
limite singulièrement la portée de celle-ci. En effet, ces grossistes situés en dehors des pavillons et du carreau exercent leur activité sous le seul régime de la liberté du commerce et de l'industrie, et imposent une concurrence aux mandataires que ceux-ci peuvent difficilement soutenir étant donné l'importance des devoirs qui leur incombent et des charges qu'ils doivent supporter et que les acteurs de ce que l'on ne tardera pas à appeler le «marché libre » (par opposition au «marché officiel») n'ont pas à subir: paiement du poids public et d'un cautionnement à la ville de Paris, limitation physique des locaux de vente par la préfecture de Police, etc.

Il apparaît donc que la principale conséquence de la loi aura été, non pas de supprimer les commerçants libres et de regrouper tous les «grossistes » sous le statut unique de mandataire, mais d'exclure les commissionnaires et négociants libres des pavillons des halles, et par-là, en un sens, de faciliter leur développement, voire leurs pratiques monopolistiques: en effet, les commissionnaires et négociants n'ont pas à respecter les heures officielles de vente auxquelles sont soumis les mandataires. Ils peuvent donc vendre avant l'ouverture légale du marché officiel, si bien que ce sont eux et non plus les mandataires qui fixent les cours; et si, pendant la journée, ils observent une tendance à la baisse, ils cessent momentanément de vendre pour reprendre après la clôture du marché officiel, lorsque l'offre se sera restreinte. Rien n'empêche non plus les commissionnaires et négociants de pratiquer les ventes successives - puisque c'est le principe de la liberté du commerce qui régit leur activité - ni même de pratiquer les ventes fictives (que la loi devait supprimer): par exemple, lorsqu'un commissionnaire n'est pas satisfait des cours du jour, il peut devenir l'espace d'un instant négociant et acheter ferme les marchandises envoyées par son expéditeur, les mettre en «resserre» et les remettre sur le marché le lendemain, réalisant une marge qu'il n'aura à partager avec personne.

\section{La prolifération d'agents «incontrôlables»}

Il importe ici de souligner avant tout que l'un des principaux obstacles à «l'application» de la loi réside dans l'attitude de la préfecture de la Seine, qui, en tant que propriétaire du marché, a le privilège d'accorder comme bon lui semble des emplacements à l'intérieur du périmètre. La contrepartie de ce statut de propriétaire est qu'elle doit financer l'entretien des halles. Or ce marché 


\section{O S S I E R}

Espaces de l'activité économique

Antoine Bernard de Raymond La construction d'un marché national des fruits et légumes: entre économie, espace et droit (I896-I995)

18. Les expéditions de fruits et légumes vers les seuls pavillons des halles centrales passent de 12125 tonnes en 1900 à 50000 tonnes en 1936 ; la consommation annuelle de fruits et légumes de la population parisienne est estimée en 1925 à 500000 tonnes (voir Conseil national économique, «Organisation du marché des fruits et légumes », 6-7 juillet 1937), soit dix fois plus que ce que le marché officiel est capable de supporter.

19. C'est moi qui souligne.

20. Le «marché libre» verra la naissance d'autres catégories moins importantes, comme les «pieds humides» ou les «terrassiers». Voir Antoine Bernard de Raymond, Des Halles centrales de Paris aux Marchés d'Intérêt National, La construction d'un marché national des fruits et légumes, DEA de sociologie, École normale supérieure (ENS)-Cachan, 2003, pp. 63-64. Le marché officiel n'échappe pas non plus à l'apparition de nouvelles fonctions non envisagées par la loi, comme les «représentants-vendeurs » dans les pavillons, et les approvisionneurs (qui ne sont pas moins de six cents en 1937) sur le carreau. lui coûte extrêmement cher. D'où la tentation, pour le préfet de la Seine, de maximiser les sommes perçues au titre du cautionnement des emplacements concédés, sans tenir compte de la «qualité» des individus bénéficiant de ces emplacements.

Ainsi, le marché «libre» prend rapidement le pas sur le marché officiel, qui, de par sa taille restreinte (les douze pavillons construits par Victor Baltard) n'est de toute façon pas à même de supporter l'augmentation de la demande en produits alimentaires de la population parisienne ${ }^{18}$. Mais, non seulement le marché libre «dépasse ( (en volumes traités aussi bien qu'en chiffre d'affaires) le marché «officiel», mais de plus, il voit apparaître de nouvelles catégories d'acteurs qui sapent les fondements même de la loi, à savoir, l'institution d'un marché représentatif des producteurs contrôlé administrativement. Une figure devient ainsi incontournable dans les halles de la première moitié $\mathrm{du} \mathrm{XX}^{\mathrm{e}}$ siècle: le répartiteur. Celui-ci reçoit de la marchandise, en général d'origine étrangère ou coloniale (mais parfois aussi métropolitaine), avec le mandat de la vendre. Mais contrairement au mandataire, il ne vend pas depuis un poste donné: il se déplace dans l'ensemble du marché (dans le marché libre comme dans le marché officiel), et tente d'écouler les produits dont il a la charge chez les uns et chez les autres. Si l'on suit la logique de la loi du 11 juin 1896, l'existence d'un tel personnage est aberrante puisqu'il a accès aux pavillons qui constituent un marché de première main et qui sont réservés aux mandataires. Là encore, certains acteurs se sont saisis de l'ambiguïté des textes régissant le fonctionnement des halles. Par une décision du 7 février 1898, l'administration du marché dispose que «les expéditions devront être accompagnées d'un bordereau collectif qui sera établi par les expéditeurs ou leurs représentants ${ }^{19}$, et qui contiendra le nom et l'adresse de ceux auxquels est destinée la marchandise». Il suffit donc au répartiteur de prétendre être le représentant de ceux qui lui ont expédié des produits; il peut ensuite lui-même déterminer la répartition du colis sur le bordereau collectif (ex post). Avec le répartiteur ce ne sont plus deux systèmes relativement imperméables qui coexistent, avec d'un côté la liberté du commerce et de l'industrie et, de l'autre, un marché encadré par des contrôles administratifs, c'est le principe de la liberté du commerce qui est réintroduit au sein même des pavillons ${ }^{20}$ ! D'autres catégories d'acteurs 
verront encore le jour ou persisteront après 1896 , comme les regrattiers (sur le carreau) ou les approvisionneurs.

Au final, on voit que les contrôles définis par la loi du 11 juin 1896 n'affectent qu'une petite partie des vendeurs des halles (au sens large) puisqu'en 1937 on compte quatre cents mandataires pour un total de près de trois mille acteurs effectuant des ventes sur le marché.

\section{Les MIN et les périmètres de protection}

À la lumière des développements qui précèdent, portant sur la phase d'élaboration ainsi que sur la portée de la loi du 11 juin 1896, il apparaît que le législateur a peutêtre manqué l'essentiel à cette occasion: la qualification institutionnelle de l'espace.

\section{Vers l'abolition de la loi du 11 juin 1896}

En effet, alors que les débats parlementaires et le texte de la loi vont se concentrer sur le statut à donner aux vendeurs, les contrôles à effectuer, les modalités de ces contrôles, ainsi que sur la répartition des compétences entre les administrations en charge de la police du marché, les questions portant sur la définition physique du marché sont laissées de côté, alors que les problèmes qu'elles soulevaient avaient été discutés par les parlementaires, comme ceux liés aux ambiguïtés de la définition des halles (la partie couverte du marché uniquement ou bien l'ensemble du périmètre), ou celui des limites du périmètre des halles centrales.

Malgré ces discussions, ces problèmes ne furent pas abordés par la loi, et «ce qui devait arriver» arriva, c'est-à-dire que le développement du marché se fit en grande partie en dehors (physiquement et juridiquement) des contrôles prévus par la loi. Se posera donc assez rapidement la question de savoir ce qu'il faut faire des commissionnaires, qui traitent à eux seuls près des trois quarts des échanges réalisés aux halles, et qui, du point de vue juridique, agissent en toute légalité, dans la zone dite du «périmètre» du marché, c'est-à-dire en dehors du périmètre de validité (du point de vue juridique) de la loi.

Le problème de la définition des halles, et plus particulièrement la définition de l'étendue et des limites du marché, a été laissé de côté, tant et si bien que des conceptions du marché différentes se font jour, entre 


\section{O S S I E R}

Espaces de l'activité économique

Antoine Bernard de Raymond La construction d'un marché national des fruits et légumes: entre économie, espace et droit (I896-I995)

\section{Voir JORF. Sénat,}

séance du 2 décembre 1937 , intervention de René Caillier, représentant de la commission du commerce. En réalité, l'argument selon lequel les mandataires fixent les cours officiels et orientent l'activité du marché «libre» n'est recevable qu'en théorie parce que les commissionnaires n'ayant pas à respecter les heures officielles d'ouverture et de clôture du marché, peuvent commencer à vendre avant les mandataires, de sorte que ce sont souvent eux, qui, dans les faits, fixent les cours.

22. Voir JORF. Sénat, séance du 2 décembre 1937. lesquelles ceux qui voudront amender ou abroger la loi de 1896 devront trancher.

Pour les représentants de la chambre de commerce de Paris par exemple, la dualité du marché parisien n'est en rien le fruit d'une mauvaise rédaction de la loi, mais est la conséquence de ce que, au contraire, la loi a fort bien organisé le marché : elle a en effet pris soin qu'une minorité de grossistes (les mandataires) - qui ont pour fonction d'harmoniser le marché en établissant des cours officiels, qui servent ensuite de référence aux commissionnaires du marché libre (qui a de toute façon la préférence des producteurs) - aient un statut spécial. Ce sont les pavillons qui constituent une exception, car la loi de l'offre et de la demande constitue une «loi naturelle» qu'on ne saurait renverser, même en légiférant. Aussi, il ne faut pas chercher à étendre le statut de mandataire au marché libre, car la vocation du marché officiel n'est pas de concentrer l'ensemble des transactions, mais d'offrir un point de repères aux commerçants libres. Aux yeux des représentants du «commerce libre», le marché officiel est pleinement justifié en ce sens qu'il joue le même rôle qu'un "phare éclairant les embarcations ${ }^{21}$ » pour qu'elles puissent s'orienter correctement, mais les contrôles plus rigoureux dont cette partie du marché fait l'objet sont totalement injustifiés si l'on croit y trouver un moyen de changer le sens du courant.

Mais cette interprétation de la loi et de la réalité est loin d'être partagée par tous. Et si la chambre de commerce condamne toute atteinte au principe de la liberté du commerce et de l'industrie, d'autres semblent penser que le principe d'égalité de tous face à la loi doit aussi avoir droit de cité, comme en témoigne cet extrait des délibérations du Conseil d'État en 1925 au sujet du règlement d'administration publique pris pour l'application de la loi de 1896, où un commissaire du gouvernement, Albert Hendlé, déclare, à propos du marché libre et du marché officiel:

«En réalité ces deux marchés n'en font qu'un [...]. Il semble donc qu'il y aurait lieu de rechercher si, en présence d'une situation de fait confondant à la fois les pavillons des halles centrales et leur pourtour, il ne conviendrait pas de considérer l'ensemble des deux marchés comme formant un marché global et d'envisager des mesures législatives permettant d'étendre à tout l'approvisionnement en gros de la ville de Paris les règlements qui, actuellement, ne peuvent s'appliquer qu'aux seules opérations effectuées aux halles centrales ${ }^{22}$.» 
L'invention des MIN: construire un marché national des fruits et légumes

Diverses tentatives d'amendement ou d'abolition de la loi du 11 juin 1896, menées avant et après la Seconde Guerre mondiale dans un cadre parlementaire et légal échoueront ${ }^{23}$, et c'est le Commissariat général du Plan qui va, à sa manière, résoudre le "problème des halles", en élaborant une réforme visant à fondre l'ensemble de la réglementation française des marchés de produits alimentaires (que ce soit à Paris ou en province) au sein d'un cadre juridique unique. Les membres du Commissariat au Plan (avec à leur tête Antoine Marès Libert Bou) cherchent ainsi à «dépasser» la loi du 11 juin 1896 de deux manières: d'une part en élaborant un cadre réglementaire qui dépasse la seule question parisienne, d'autre part, en tirant les leçons des «échecs» de la loi de 1896, en définissant clairement les frontières des marchés de gros. Cette réforme avait pour but de rationaliser le commerce des produits alimentaires en France en unifiant le marché au niveau national. Concrètement, il s'agissait de bâtir un réseau de marchés physiques modernes connectés entre eux par un système des nouvelles du marché afin de permettre une orientation adéquate des flux de marchandises et une fixation rationnelle des cours.

En effet, pour les fonctionnaires du Plan, c'est l'incertitude du producteur ou de l'expéditeur au moment de mettre en marché les denrées qu'il détient qui cause les maux que connaît le marché français au sortir de la Seconde Guerre mondiale. C'est cette incertitude qui permet la prolifération d'intermédiaires inutiles et de circuits parallèles, ainsi que la fraude et les pratiques monopolistiques. Ce sont encore l'incertitude et le manque d'information qui engendrent des disparités régionales: au même moment, un marché de province peut être mal approvisionné et les prix y atteindre des sommets, alors que Paris va connaître la pléthore et que les cours vont s'y effondrer ${ }^{24}$.

Le manque d'information est donc jugé responsable du cloisonnement des différents circuits d'achalandage, limitant de ce fait la concurrence. Il s'agit donc de supprimer ces zones d'ombres qui empêchent le producteur de tirer le meilleur parti de son travail et constituent une tentation perpétuelle pour les intermédiaires. Autrement dit, on cherche à instaurer un fonctionnement véritablement marchand en rendant accessible à tous une information claire
23. Voir A. Bernard de Raymond, Des Halles centrales..., op. cit., pp. 70-85.

24. En effet, l'incertitude qui plane sur le devenir des fruits de leur travail fait que le choix d'orienter systématiquement leurs produits vers la capitale représente une solution rationnelle pour les cultivateurs, dans la mesure où l'on est à peu près certain que ces marchandises y trouveront preneur, quitte à ce qu'elles soient en suite réexpédiées vers la province. Voir Daniel Barrère, Le marché français des fruits et légumes, Paris, M.-T. Génin, 1954, p. 20. 


\section{O S S I E R}

Espaces de l'activité économique

Antoine Bernard de Raymond La construction d'un marché national des fruits et légumes: entre économie, espace et droit (I896-I995)

25. Avis du Conseil économique, dans sa séance du 8 octobre 1952.

26. Commission d'étude des marchés gares, «Les marchés gares et la réorganisation de la commercialisation des fruits et légumes », rapport remis au président du Conseil, juin 1953. et précise sur l'état du marché. Certains font ainsi observer que «la loi de l'offre et de la demande ne s'applique d'une façon correcte que si les quantités apportées sur le marché ne sont pas artificiellement déterminées ${ }^{25}$ ».

La réforme qui est proposée par le $\mathrm{Plan}^{26}$ a donc pour but une mise en concurrence des différents circuits de distribution, afin de supprimer les zones d'incertitude et les rentes de situation. L'instrument de cette mise en concurrence doit être la construction de marchés physiques modernes (dotés d'infrastructures logistiques importantes et reliés au rail et à la route) où sera concentré l'ensemble de l'offre et de la demande. De cette concentration résultera une confrontation rationnelle de l'offre et de la demande et donc une baisse des prix de gros, ainsi qu'une sélection des éléments les plus performants du commerce de gros en fruits et légumes; on devra ainsi aboutir à une

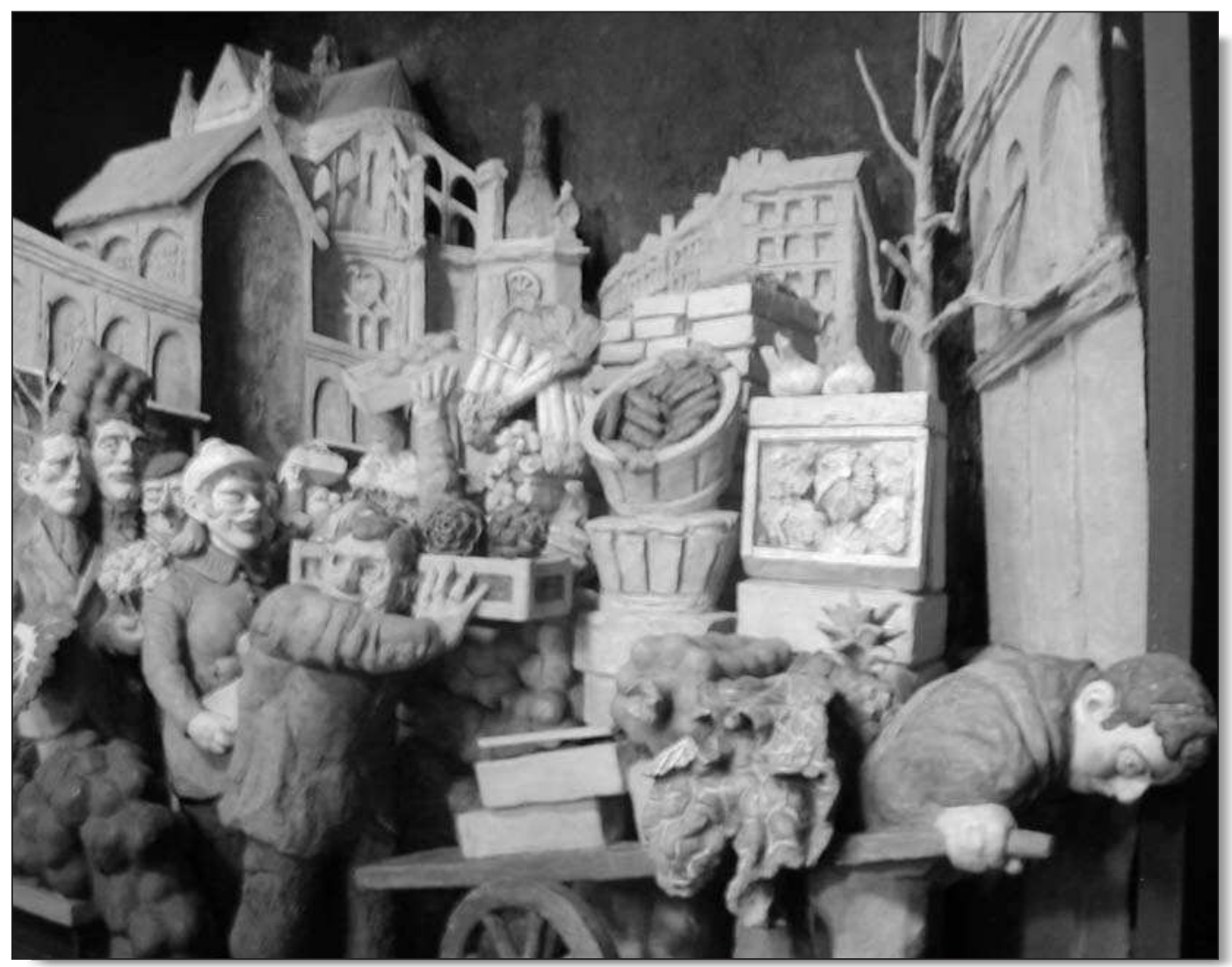

Les " gens des Halles » quittent Paris, mais tous ne trouveront pas forcément une place au sein des nouveaux réseaux de distribution modernes que sont les MIN.

Le départ des fruits et légumes du cœur de Paris le 28 février 1969, sculpture de Raymond Mason, église Saint-Eustache. () Cliché Antoine Bernard de Raymond. 
modernisation, une concentration et une homogénéisation des entreprises pratiquant le commerce de gros de fruits et légumes, et, in fine, à une baisse des prix de détails.

Le rapport du Plan est repris par le gouvernement Laniel, par le décret 53-959 du 30 septembre $1953^{27}$, qui constitue l'acte de naissance des MIN. Mais, ce décret ne contient pratiquement pas de mesures concrètes d'organisation du marché des fruits et légumes. Le contenu concret de cette réforme ne sera élaboré que très progressivement, entre 1958 et $1967^{28}$.

Pour le point de vue qui est le nôtre, à savoir celui de la qualification institutionnelle de l'espace, on ne retiendra de ce lent «travail législatif» que la réglementation sur les périmètres de protection: en effet, la volonté de concentrer l'offre et la demande en des lieux précis pour soumettre les grossistes à un cadre réglementaire unique et généraliser la concurrence impliquait de prendre des mesures pour empêcher que, comme dans les cas des halles centrales de Paris, des agents économiques s'installent à proximité du marché pour profiter des avantages qu'il offre sans en subir les inconvénients (charges financières, etc.). C'est pourquoi les MIN furent dotés de périmètres de protection, zones englobant le marché, mais beaucoup plus vastes que lui, à l'intérieur desquels les ventes en gros des produits échangés sur le MIN sont interdites. Plus précisément, il existe deux sortes de périmètres de protection: le périmètre de protection positif, à l'intérieur duquel les transactions «autres que de détail» sont prohibées; le périmètre de protection négatif, à l'intérieur duquel la création, l'extension ou le déplacement d'établissements pratiquant la vente en gros de produits échangés sur le MIN sont interdits. Il fut généralement recouru au périmètre de protection négatif, et seuls les MIN de Paris (Rungis), Bordeaux et Strasbourg furent dotés d'un périmètre positif.

L'architecture et l'agencement matèriel des pavillons sont censés servir le projet de mise en concurrence de tous les circuits de distribution en «clarifiant» l'offre et en facilitant la circulation des personnes, les acheteurs pouvant désormais d'un seul coup d'œil évaluer l'état du marché.

\section{Une institution sous tension}

Très vite - et, dans certains cas, dès avant l'ouverture du marché - les gestionnaires des MIN vont être confrontés à des tensions résultant non seulement de la disparité des acteurs installés sur ces marchés, mais aussi du
27. Le recours au décret (plutôt qu'à la loi) permet au gouvernement d'éviter une discussion parlementaire longue et susceptible de limiter singulièrement la portée de la loi, voire d'en empêcher purement et simplement le vote, comme en témoignent les échecs de toutes les tentatives d'amendement de la loi du 11 juin 1896 dans un cadre parlementaire dans les années 1930 et 1940.

28. Aujourd'hui, les textes en vigueur réglementant l'organisation et le fonctionnement des MIN sont l'ordonnance 67-808 du 22 septembre 1967 et les décrets 68-658 à 68-661 du 10 juillet 1968 pris pour son application. 


\section{O S S I E R}

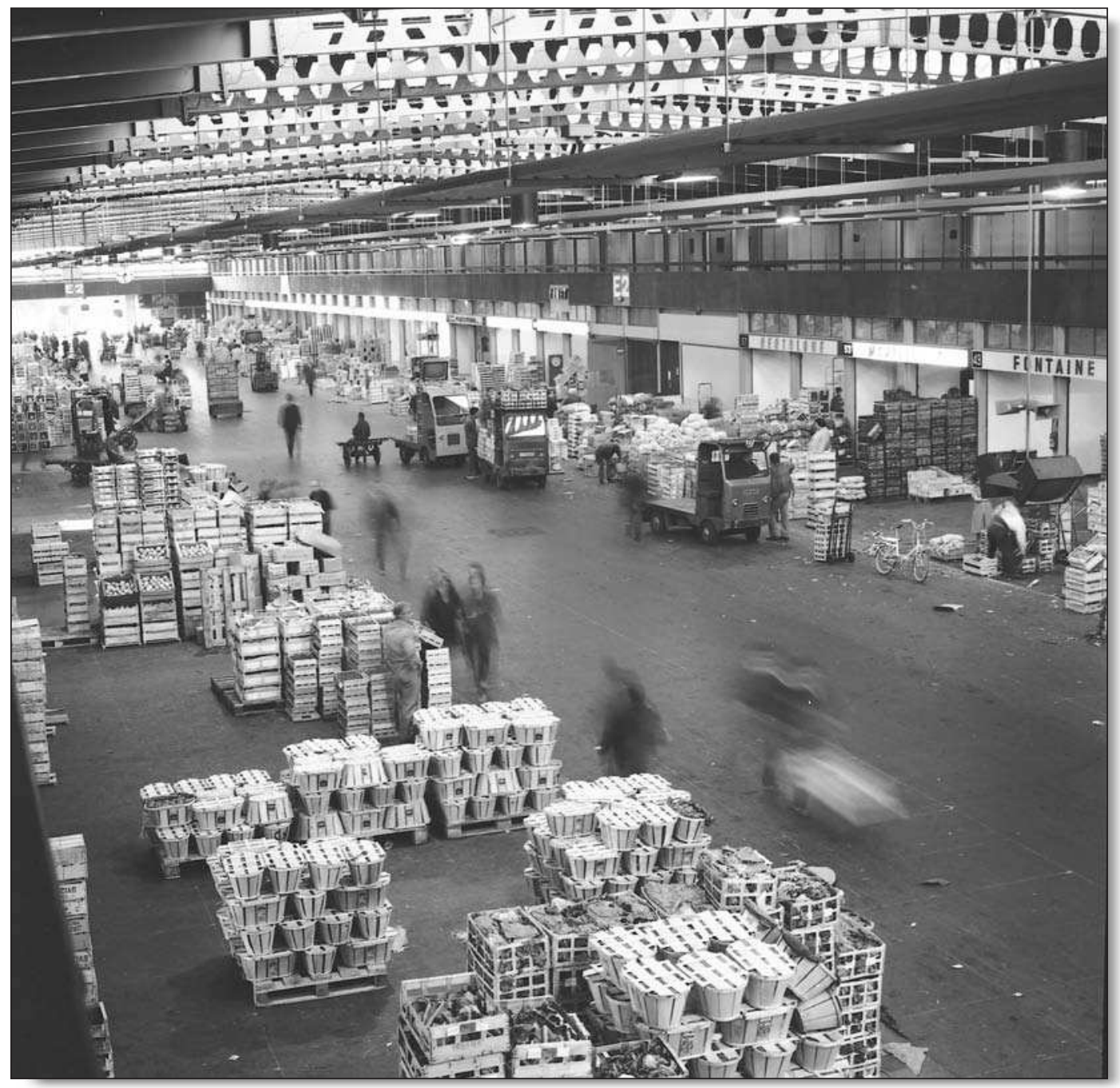

Un pavillon des fruits et légumes en activité, mars I969. () Photo SEMMARIS, Archives départementales du Val-de-Marne, 2447 W I247.

développement très rapide de nouveaux acteurs économiques en dehors des marchés.

On trouve schématiquement deux types d'entreprises sur les MIN: les grossistes «de carreau» (ou traditionnels) et les grossistes «à service complet» (ou gasc). L'activité économique des premiers se caractérise par la vente de gré à gré en présence de la marchandise possiblement échangée. Acheteurs et demandeurs se font face et négocient le prix des produits tout en évaluant leur qualité (par une investigation sensorielle notamment). Les gasc, eux, livrent les produits qu'ils commercialisent. 
De ce fait ils réalisent en principe leurs ventes à distance, et leur politique commerciale repose sur la normalisation des produits (censée garantir la qualité des biens offerts). Potentiellement, les gasc peuvent tout à fait se passer de l'existence de marchés physiques, et ont surtout besoin de disposer de plates-formes logistiques performantes. À l'inverse, l'activité de chaque grossiste de carreau considéré individuellement ne peut en réalité se concevoir, ou du moins perd une partie de sa signification (et de sa rentabilité), sans la présence d'autres grossistes de carreau effectuant la même activité. Plus précisément, l'activité d'un grossiste de carreau ne peut se concevoir sans le principe de marché, qui donne sa valeur à cette activité29. C'est particulièrement vrai pour ceux d'entre les grossistes de carreau qui sont spécialisés dans un type de produit.

L'approvisionnement par entrepôt des points de vente au détail s'est progressivement développé depuis les années 1960, sa forme la plus extrême étant la «grande distribution» qui a pour principe l'approvisionnement de chaînes de magasins par des entrepôts relevant de la même société, avec pour politique de supprimer au maximum les intermédiaires et de construire des relations directes avec des producteurs et expéditeurs, qui sont référencés par les centrales d'achat du groupe et régulièrement mis en concurrence entre eux.

Alors que les grossistes «traditionnels» n'ont pour ainsi dire pas le choix, et sont par la force des choses contraints à s'installer sur un MIN (puisque leur activité ne peut se penser sans l'existence de marchés physiques), les agents pratiquant le commerce à distance et dématérialisé (les gasc, le commerce intégré) peuvent adopter trois stratégies vis-à-vis des MIN: ou bien ils s'installent sur le marché, considérant que malgré les charges que cela fait peser sur leur activité, cela permet de toujours disposer de sources d'approvisionnement sûres et de profiter des courants commerciaux qui y sont drainés, ou bien, choisissant de tirer profit des ressources de l'industrialisation et de leur «atopisme», se fixent en dehors des MIN et de leurs périmètres de protection, soit enfin ils peuvent demander (en principe) une dérogation pour s'établir à l'intérieur de la zone de protection du MIN.

Il est prévu dans l'ordonnance du 22 septembre 1967 (article 8) que des dérogations aux interdictions liées aux périmètres de protection (articles 5, 6 et 7) pourront être accordées à titre exceptionnel par les ministres de tutelle
29. Voir Antoine Bernard de Raymond, «Le marché aux fruits et légumes de Rungis», Terrains \& travaux, $\mathrm{n}^{\circ} 4$, 2003, pp. 82-110. 


\section{O S S I E R}

Espaces de l'activité économique

Antoine Bernard de Raymond La construction d'un marché national des fruits et légumes: entre économie, espace et droit (I896-1995)
30. Jean-Paul Montenot et Michel-Alexandre Rahmani, MIN d'aujourd'hui, marchés de demain, Paris, Centre technique interprofessionnel des fruits et légumes, 1992, p. 27. des MIN. Ces dérogations doivent être de nature à améliorer la productivité de la distribution (décret n ${ }^{\circ}$ 68-660 du 10 juillet 1968, article 2).

Compte tenu de la volonté affichée par les pouvoirs publics de concentrer l'offre et la demande sur les MIN afin de «clarifier» les transactions, on aurait pu penser que les articles consacrés aux dérogations seraient «appliqués» de manière très stricte et que le nombre d'autorisations d'implantation à l'intérieur des périmètres délivrées aurait été limité.

En réalité il n'en fut rien et la période qui va de la fin des années 1960 au début des années 1980 se caractérise par des dérogations accordées «facilement» (par les préfets ou bien par le comité de tutelle des MIN) sans qu'on puisse déceler une logique précise dans ces décisions. Par exemple, deux juristes, dans une étude réalisée pour l'Union nationale du commerce de gros en fruits et légumes (UNCGFL), ont

«[...] constaté que l'administration ne suivait pas de ligne directrice et semblait accorder ces dérogations de façon quelque peu désordonnée. [...] Il est d'ailleurs symptomatique de constater que depuis 1980, le Comité de Tutelle est incapable de fournir le détail des dérogations et motifs retenus; il n'existe aucun tableau d'octroi de dérogations $»^{30}$.

Les grossistes des MIN ne se sont dans un premier temps pas vraiment souciés de la prolifération d'entreprises de gros à l'intérieur des périmètres de protection, persuadés qu'ils étaient d'être indispensables au fonctionnement de la filière, notamment aux groupes de grande distribution.

Mais progressivement, à partir du milieu des années 1980, au vu de la diminution très rapide du nombre d'entreprises de gros sur les MIN que ce soit pour cause de faillite, ou de non-reprise de l'affaire suite à un départ à la retraite, les usagers des MIN ont progressivement pris conscience de la fragilité de leur situation économique, et du caractère très relatif de leur utilité vis-à-vis des acteurs dominants de la filière qu'étaient devenus les groupes de «grande distribution». Les syndicats de grossistes notamment ont entrepris des démarches visant à susciter une «prise de conscience» de la part de leurs adhérents, comme en témoigne la publication de l'étude déjà citée MIN d'aujourd'hui, marchés de demain en 1992 (pour le compte de l'UNCGFL). Cette étude va stigmatiser, comme on l'a vu, l'absence de logique dans l'octroi des dérogations aux interdictions résultant des périmètres de 
protection, et l'absence de visibilité du comité de tutelle, chargé de statuer sur les demandes de dérogation:

«L'octroi de ces dérogations a largement contribué au malaise aujourd'hui ressenti sur les MIN. Nombre d'usagers vont découvrir l'existence du Comité de Tutelle avec la présente consultation ce qui est assez symptomatique de l'assistance qui a existé entre le tuteur et «ses enfants» depuis plus de 20 ans $^{31}$.»

Ainsi les grossistes ont progressivement découvert la législation sur les périmètres de protection, la façon dont elle avait jusqu'alors été «appliquée» et les menaces que cela faisait peser sur leur avenir. Du coup, les années 1990 semblent marquer le début d'une période de vigilance accrue de la part des syndicats de grossistes vis-à-vis des dérogations accordées par le comité de tutelle, vigilance pouvant aller jusqu'à la contestation devant juridiction de la légalité de la dérogation. Ajoutons que cette découverte de la législation et cette "politique de vigilance» vont s'accompagner, de la part des représentants des professionnels, d'une tentative de redéfinition de la fonction des MIN et de leurs usagers. L'UNCGFL, notamment, tente d'engager ses adhérents vers une différenciation et une segmentation accrues de leurs activités et de leurs produits $^{32}$. Elle engage ainsi ses membres à faire certifier leurs compétences (en utilisant les normes ISO - International Organization for Standardization - ou des cahiers des charges) et à faire reconnaître la spécificité de leurs produits (en utilisant des labels, des AOC - Appelation d'origine contrôlée - etc.). Les MIN se trouvent ainsi détournés de leur mission initiale de concentration et d'homogénéisation de la filière pour devenir des espaces de segmentation du marché et de protection des pans «traditionnels» de la filière.

Progressivement les grossistes en viennent à concevoir les MIN (compris à la fois comme instruments de travail et comme concept juridique) comme un moyen de se défendre contre le commerce intégré qui favorise leur élimination de la filière. À cet égard, on peut noter que la jurisprudence retient deux décisions de justice assez proches, qui impliquent à chaque fois la société Metro: la dérogation dont elle a bénéficié pour s'installer à l'intérieur du périmètre de protection d'un MIN a été attaquée dans un premier temps (et avec succès) par les usagers de ce marché ou par une collectivité locale. Mais ces annulations de dérogations sont ensuite contestées, soit par le comité de tutelle, soit par la société bénéficiaire (Metro) devant le Conseil d'État.
31. Ibid.

32. Antoine Bernard de Raymond, Le marché aux fruits et légumes de Rungis, mémoire de maîtrise de sociologie, université de Nanterre, ENS-Cachan, 2001. 


\section{O S S I E R}

Espaces de l'activité économique

Antoine Bernard de Raymond La construction d'un marché national des fruits et légumes: entre économie, espace et droit (I896-1995)
33. Conseil d'État (CE), Section du contentieux, $n^{\circ} 71.573$, 10 juillet 1987.
Ces décisions font une nouvelle fois clairement ressortir les motivations des grossistes vis-à-vis des périmètres de protection, à savoir la protection contre la «concurrence de porte». En effet, du point de vue d'un grossiste installé sur un MIN, l'installation d'un concurrent à proximité du marché constitue une distorsion de concurrence, ledit concurrent pouvant profiter des mêmes avantages commerciaux que lui (les flux drainés par le marchés) sans en supporter les inconvénients (paiement de la redevance, difficulté à adapter ses locaux à ses besoins).

Le comité de tutelle et la société Metro avancent principalement deux moyens qui visent à montrer que l'octroi de la dérogation était suffisamment motivé: d'une part l'autorité qui a accordé la dérogation a estimé que l'installation de Metro était de nature à «améliorer la productivité de la distribution", ce en quoi la dérogation circonvenait aux conditions d'octroi définies à l'article 2 du décret du 10 juillet 1968; d'autre part, dans les deux cas, l'octroi de la dérogation avait été subordonné à l'adjonction d'une clause d'approvisionnement préférentiel, conformément à l'article 6 du décret du 10 juillet 1968 qui précise que le comité de tutelle, ou, le cas échéant, le préfet par délégation, fixe les conditions auxquelles il accorde la dérogation et

«[...] peut en subordonner l'octroi à la conclusion entre le gestionnaire du marché et le bénéficiaire d'une convention par lequel ce dernier s'engage [...] à observer certaines obligations relatives, notamment, aux horaires de vente, au contrôle des transactions, au recensement des quantités et des prix».

La dérogation accordée à Metro à Bordeaux prévoit ainsi que «la société Metro s'approvisionnera à hauteur de $80 \%$ auprès des concessionnaires dudit marché et qu'elle limitera à $350 \mathrm{~m}^{2}$ la surface de vente des produits

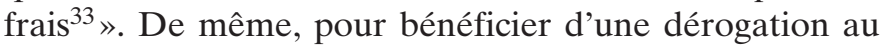
sein du périmètre de protection du MIN de Montpellier, Metro avait dû passer une convention avec la société gestionnaire du MIN par laquelle elle s'obligeait à réaliser $70 \%$ au moins de ses achats de fruits et légumes frais sur le MIN de Montpellier.

Dans les deux cas, le jugement qui annulait la dérogation est cassé par le Conseil d'État, qui estime par exemple, dans le cas du MIN de Montpellier que:

«Tant du fait de cette clause d'approvisionnement préférentiel, propre à favoriser l'apport d'une clientèle nouvelle au marché d'intérêt national, dont l'activité, concurrencée par celle de magasins en gros de grande surface implantés en 
dehors du périmètre protégé, se caractérisait par une baisse de volumes des affaires traitées, que des avantages devant découler pour les professionnels de l'alimentation de la gamme étendue de produits offerts par l'établissement de la société Metro Libre Service de gros de Montpellier, de ses larges horaires ouverture et de ses conditions et méthodes particulières de vente et de délivrance des marchandises, le préfet [...] n'a pas commis d'erreur manifeste d'appréciation en estimant que l'octroi à cette société de la dérogation qu'elle avait sollicitée [...] serait de nature à améliorer la productivité de la distribution locale en gros des fruits et légumes frais ${ }^{34}$.»

Ces deux décisions du Conseil d'État ont deux conséquences manifestes: d'une part elles rejettent une interprétation possible de l'architecture légale des MIN, celle qui a pu être à un certain moment adoptée par les usagers des MIN (et dans les années 1960, mais pour la dénoncer, par les tenants du commerce libre) qui consiste à voir dans les textes sur les périmètres de protection l'outil de constitution d'un monopole au bénéfice des MIN; d'autre part, en faisant des «clauses d'approvisionnement préférentiel» une condition de validation de la dérogation, le Conseil d'État constitue le MIN comme un espace à part, qu'il faut protéger et entretenir. Le motif de ce choix apparaît de façon manifeste lorsqu'il est souligné que cette clause d'approvisionnement préférentiel permettra d'apporter une clientèle nouvelle au MIN (c'est-à-dire aux grossistes du MIN) dont l'activité est concurrencée par celle de magasins en gros de grande surface implantés en dehors du périmètre protégé. Le jugement distingue donc clairement deux formes de commerce, dont l'une, la plus traditionnelle, et qui prend corps dans les MIN, doit être défendue contre l'autre.

Ainsi ces deux décisions (publiées au recueil Lebon) tendent à faire des MIN et de leur(s) périmètre(s) de protection des «parcs naturels» du commerce de gros des produits alimentaires: on y protège les espèces en voie de disparition. On interdit de «chasser» dans un espace donné pour préserver la «diversité biologique» du commerce.

Il apparaît alors que l'espace n'est pas une donnée purement matérielle qui pourrait se résumer à la mesure des distances physiques. Au contraire, le rôle de l'espace dans l'activité économique ne peut se comprendre que si l'on conçoit l'espace comme une construction sociale, en l'occurrence une construction institutionnelle. Les stratégies de localisation (voire les stratégies économiques tout
34. CE, Section du contentieux, $\mathrm{n}^{\circ} 140.408, \mathrm{n}^{\circ} 141.049,14$ juin 1995. 


\section{O S S I E R}

Espaces de l'activité économique

Antoine Bernard de Raymond La construction d'un marché national des fruits et légumes: entre économie, espace et droit (I896-1995)

35. Ainsi, à propos des halles au Moyen Âge on peut lire: «Quelles étaient les limites des halles, de ce marché [...] où, à certains jours de la semaine se concentrait le commerce de la capitale? Il ne semble pas qu'aucun texte les ait jamais fixées avec précision» (Jean Martineau, Les Halles de Paris, des origines à 1789 , Paris, Montchrestien, 1960, p. 62). court) des acteurs du commerce de gros de fruits et légumes sont vouées à rester inaccessibles au chercheur si celui-ci ne tient pas compte de la définition légale et judiciaire de l'espace. L'incertitude qui plane de manière chronique ${ }^{35}$ sur les frontières physiques du marché, et sur la délimitation des différentes zones (halle, carreau, etc.) à l'intérieur du marché, permet à un grand nombre de commerçants de s'installer à proximité des halles centrales pour profiter des flux commerciaux qu'elles drainent sans en subir les inconvénients (paiement de droits d'abri, contrôles administratifs, etc.). Ainsi, la loi du 11 juin 1896, introduisant de facto une bipartition du marché, échoue à créer une catégorie unique (celle de «mandataire») d'agents opérant uniquement au nom de producteurs et d'expéditeurs de province qu'ils représentent. Comme rien n'empêche les commissionnaires du «marché libre» de pratiquer le commerce pour leur propre compte, les transactions successives sur le marché peuvent avoir lieu «en toute impunité », et ces pratiques sécrètent de nouvelles catégories d'acteurs (répartiteurs, regrattiers, pieds humides, terrassiers, etc.) qui n'ont souvent d'autre fonction que de faire circuler la marchandise à l'intérieur des halles.

Ce sont donc des dispositions de droit et leur activation qui nous permettent de comprendre les stratégies économiques développées par les uns et les autres. Pour éviter que se posent les mêmes problèmes que lors de la mise en œuvre de la loi de 1896, les pouvoirs publics, lorsqu'ils inventent les MIN, dotent ces marchés de périmètres de protection afin de soumettre l'ensemble des grossistes à la même réglementation. Mais une fois encore, la construction judiciaire de l'espace différera sensiblement de celle opérée par le droit légiféré. Le contentieux mobilisant la législation sur les périmètres de protection nous fait voir les motivations de nouveaux commerçants capables de se passer des marchés physiques pour leur activité livrer une «concurrence de porte» aux MIN. Face à cette situation, les arrêts du Conseil d'État dans les années 1990 tendent à faire des MIN non plus les lieux de mise en concurrence généralisée de l'ensemble des entreprises de gros en produits alimentaires, mais des espaces de protection du commerce traditionnel. 